\begin{abstract}
Cohomology and deformation theories are developed for Poisson algebras starting with the more general concept of a Leibniz pair, namely of an associative algebra $A$ together with a Lie algebra $L$ mapped into the derivations of A. A bicomplex (with both Hochschild and Chevalley-Eilenberg cohomologies) is essential.
\end{abstract}

\title{
COHOMOLOGY AND DEFORMATION OF LEIBNIZ PAIRS
}

\author{
M. FLATO, M. GERSTENHABER, AND A. A. VORONOV
}

December 26, 1994

The importance of Poisson algebras in classical mechanics makes it useful to have a deformation theory for them. To construct this we are led to define a more general concept: A Leibniz pair $(A, L)$ consists of an associative algebra $A$ and a Lie algebra $L$ over some common coefficient ring $k$, connected by a Lie algebra morphism $\mu: L \rightarrow$ Der $A$, the Lie algebra of $k$-linear derivations of $A$ into itself. It can happen that $L$ is identical with $A$ as a $k$-module; but note that in the graded case discussed below the gradings may differ. When this is so, denoting the associative product of $a, b \in A$ by $a b$ and their Lie product by $[a, b]$, if one has further that $\mu(a)(b c)=[a, b c]=$ $[a, b] c+b[a, c]$, then $A$ will be called a non-commutative Poisson algebra ("ncPa"). A Poisson algebra in the usual sense is one where the associative multiplication on $A$ is commutative (and $k=\mathbb{R}$ or $\mathbb{C}$ ). A Leibniz pair with $A=L$ need not be a Poisson algebra since one may have, for example, an abelian Lie multiplication together with a non-trivial structure morphism $\mu$.

This notion of a non-commutative Poisson algebra is a particular case of that suggested by $\mathrm{P} . \mathrm{Xu}[21]$. In his definition, he considers an associative algebra $A$ together with a class $\Pi \in H^{2}(A, A)$ such that $[\Pi, \Pi]=0$ in $H^{3}(A, A)$ in the sense of the Gerstenhaber bracket on Hochschild cohomology of $A$. Xu's definition is especially suitable for the geometric situation, such as $A=\mathbb{C}\left[x_{1}, \ldots, x_{n}\right]$ or $A=C^{\infty}(M)$, when $M$ is a manifold and one takes multilinear differential operators as cochains. Then $H^{2}(A, A)$ will be naturally isomorphic to the space of bivector fields and the condition $[\Pi, \Pi]=d B$, where, due Hodge theory for Hochschild cohomology, one can assume $B$ is symmetric; this will yield the Jacobi identity for $\Pi$. Thus, $\Pi$ will give rise to a Poisson bracket on $A$. For a general commutative or noncommutative algebra $A$, $H^{2}(A, A)$ would not be exhausted by skew biderivations of $A$, and the relevance of a class $\Pi$ to any kind of Poisson bracket on $A$ would be lost. Our definition translates

The second author wishes to thank the U.S. National Security Agency for partial support during the preparation of this paper

The third author wishes to thank the National Science Foundation for partial support during the preparation of this paper 
into this language as follows. A non-commutative Poisson algebra is nothing but an associative algebra $A$ and a skew biderivation $\Pi \in C^{2}(A, A)$ satisfying the Jacobi identity. It will not even automatically be a 2-cocycle, unless $A$ is commutative.

A Leibniz pair $(A, L)$ in which $A$ is commutative, $L$ is an $A$-module, and the morphism $\mu: L \rightarrow$ Der $A$ is an $A$-module morphism will be called a Rinehart pair, $[19,18,16]$, provided abbreviating

$$
\mu(x) a=\mu(x, a)=[x, a]: L \otimes A \rightarrow A,
$$

with $x \in L, a \in A$, the following natural condition is also satisfied

$$
[x, a y]=[x, a] y+a[x, y] .
$$

Here the commutativity of $A$ as an associative algebra is essential, for it is only in this case that the derivations of $A$ form a module over $A$. Any commutative Poisson algebra $A$ gives rise to a Rinehart pair $(A, A)$ but one must be careful in notation: writing ad $b$ for the mapping sending $c$ to $[b, c]$ one must distinguish between $a$ ad $b$ and ad $a b$.

Leibniz pairs $(A, L)$ are common but the concept seems not to have been previously considered in this generality. The simplest example is that where $A$ is an arbitrary associative algebra and $L$ is just Der $A$ or $A$ itself, considered as the Lie algebra of inner derivations of $A$. This includes the case where $A$ is either an ordinary polynomial ring $k\left[x_{1}, \ldots, x_{n}\right]$ or free non-commutative polynomial ring $k\left\langle x_{1}, \ldots, x_{n}\right\rangle$ in $n$ variables and $L$ the "linear first-order differential operators", i.e., the free $k$ module spanned by $\partial / \partial x_{1}, \ldots, \partial / \partial x_{n}$. (In the second case $A$ is identical with the tensor algebra over $k$ on the module spanned over $k$ by the variables).

A most important example of a commutative Leibniz pair is $\left(C^{\infty}(\mathcal{M})\right.$, Vect $\left.\mathcal{M}\right)$, where $A$ is the algebra of smooth functions on a manifold $\mathcal{M}$ and $L$ the Lie algebra of smooth vector fields; this is a Rinehart pair. Of special interest is the case when $\mathcal{M}$ is symplectic or Poisson (for a recent monograph on Poisson manifolds see [20]), $A=C^{\infty}(\mathcal{M})$ and $L=A$ endowed with the Poisson bracket (central extension by the constants of the Lie algebra of Hamiltonian vector fields); there the theory of star products [1], associative deformations of $A$ giving rise to Lie algebra deformations of $L$, is a prototype of the theory we are developing here; in particular the interplay between the (infinite) Hochschild cohomology of $A$ and the (finite) Chevalley-Eilenberg cohomology of $L$ was essential there, and is in fact one of the main motivations for introducing Leibniz pairs and Poisson algebras.

Leibniz pairs also arise whenever a Lie group $G$ operates as a group of automorphisms on an associative algebra $A$; the pair is then $(A, \mathfrak{g})$, where $\mathfrak{g}=\operatorname{Lie} G$, the Lie algebra of $G$. (One may view the Lie group as acting on a "non-commutative function space" $A$.) This situation occurs frequently in mathematical physics when $A$ is a $C^{*}$ algebra of observables (crossed products of a $C^{*}$ algebra and a group of automorphisms), including the so-called theory of KMS states (generalized equilibrium 
states) where $\mathfrak{g}$ is one-dimensional, generating a semi-group. A special case arises in geometry when a Lie group $G$ acts on a manifold $\mathcal{M}$. The pair is then $\left(C^{\infty}(\mathcal{M}), \mathfrak{g}\right)$.

Analogous to the "canonical" Leibniz pair $(A$, Der $A)$ associated to an associative algebra $A$, if one has a Lie algebra $L$ then one can form the Leibniz pair $(\mathcal{U} L, L)$, where $\mathcal{U} L$ is the universal enveloping algebra of $L$.

Another interesting example arises in the context of quantum groups at roots of unity. Suppose $\mathcal{U}_{q}(\mathfrak{g})$ is a quantum group. Specializing $q$ to a root $\epsilon$ of 1 , one usually gets a big center $Z$. The center is then a Poisson algebra with respect to the bracket

$$
\{x, y\}=\lim _{q \rightarrow \epsilon} \frac{[\tilde{x}, \tilde{y}]}{q-\epsilon},
$$

where $\tilde{x}$ and $\tilde{y} \in \mathcal{U}_{q}(\mathfrak{g})$ denote liftings of elements $x$ and $y \in Z$. This bracket can be extended to an action of $Z$ on $\mathcal{U}_{\epsilon}(\mathfrak{g})$ by derivations, and thus $\left(\mathcal{U}_{\epsilon}(\mathfrak{g}), Z\right)$ is another example of a Leibniz pair.

In this paper we develop cohomology and deformation theories for Leibniz pairs, Rinehart pairs, ncPa's, and Poisson algebras. For the latter it generally will not matter if a Poisson algebra is commutative or not, so "Poisson algebra" will henceforth include the non-commutative case unless it is said otherwise. The theory will be written in an algebraic context, but its extension to a topological context in the infinite case (at least for Fréchet nuclear algebras, as in [2]) is straightforward.

As observed a Leibniz pair may be viewed as an infinitesimal version of an algebra with a group of operators. The deformation theory of such objects is clear if the group is kept fixed [11]. It seems less natural, however, to consider simultaneous deformation of the algebra (in some sense, a local object) together with that of the group (a global one). Passage to the Leibniz pair (which consists of two local objects) may be a remedy. In suitable cases the passage from an algebra with operators to a Leibniz pair $(A, L)$ can be reversed, for example, when $A$ and $L$ are both finitedimensional (real or complex), the adjoint group of $L$ then operates on $A$.

\section{Note ON THE GRADED CASE AND OPERADS}

While we do not explicitly consider the graded case here, our methods remain applicable. An important open question in both the ungraded and graded cases is the relationship between our cohomology theory and that constructed by other methods. Poisson algebras are special cases of operad algebras, cf. [15], for which there is already a general cohomology theory. Further Getzler and Jones [14] have studied a cohomology theory for Gerstenhaber algebras, one of the two natural graded generalizations of Poisson algebras, which we will describe in this Section. In the more general context of algebras with many operations grouped into dot-like and bracket-like ones, satisfying some distributivity laws, Fox and Markl [6] have studied cohomology using the operad cohomology viewpoint as well. Fox and Markl show that for such algebras, the complex computing cohomology is the total complex of 
a bicomplex, cf. Section 5 below. Similar considerations are found in the cyclic cohomology bicomplex [4], where also one direction is Hochschild cohomology and which classifies the so-called [5] closed star products (for which a trace can be defined in the star product algebra).

We consider first graded Leibniz pairs $(A, L)$. Here both $A$ and $L$ are graded, degrees of elements being indicated by superscripts, and $L$ is a graded Lie algebra, cf. [7]. That is,

$$
\begin{gathered}
{\left[x^{m}, y^{n}\right]=-(-1)^{m n}\left[y^{n}, x^{m}\right] \text { and }} \\
(-1)^{m p}\left[x^{m},\left[y^{n}, z^{p}\right]\right]+(-1)^{n m}\left[y^{n},\left[z^{p}, x^{m}\right]\right]+(-1)^{p n}\left[z^{p},\left[x^{m}, y^{n}\right]\right]=0 .
\end{gathered}
$$

The first equation asserts that the multiplication in $L$ is "graded skew", the second is the graded Jacobi identity. Such $L$ are often called "Lie superalgebras." A graded derivation of degree $p$ of $A$ is a linear map $\mathcal{D}: A \rightarrow A$ with $\mathcal{D}\left(A^{m}\right) \subset A^{m+p}$, (where $A^{m}$ denotes the homogeneous part of $A$ of degree $m$ ) and

$$
\mathcal{D}\left(a^{m} b^{n}\right)=\left(\mathcal{D} a^{m}\right) b^{n}+(-1)^{p m} a^{m}\left(\mathcal{D} b^{n}\right) .
$$

These form a graded Lie algebra, and the structure morphism $\mu: L \rightarrow \operatorname{Der} A$ is now required to be a morphism of graded Lie algebras, hence to carry $z^{p}$ to a homogeneous derivation of $A$ of degree $p$.

The associative algebra $A$ is called graded commutative or "supercommutative" if $a^{m} b^{n}=(-1)^{m n} b^{n} a^{m}$, as, for example, in a Grassmann algebra. There are now two significantly different generalizations of an ordinary Poisson algebra, both of which have their "right" and "left" versions.

In a right "Poisson" superalgebra, $A$ is simultaneously a graded commutative algebra and a graded Lie algebra with the same grading, and right Lie multiplication by $c^{p}$ (i.e., the operation carrying $a$ to $\left[a, c^{p}\right]$ ) is a graded derivation of degree $p$. That is, we have $\left[a^{m} b^{n}, c^{p}\right]=\left[a^{m}, c^{p}\right] b^{n}+(-1)^{m p} a^{m}\left[b^{n}, c^{p}\right]$. In the left version, it is left Lie multiplication by $c^{p}$ which is a graded derivation of degree $p$, i.e., we have $\left[c^{p}, a^{m} b^{n}\right]=\left[c^{p}, a^{m}\right] b^{n}+(-1)^{m p} a^{m}\left[c^{p}, b^{n}\right]$. There is no significant difference between these, since one can pass from one to the other by replacing the associative multiplication in $A$ by its "opposite", i.e., by reversing its order, but keeping the Lie operation unchanged. A right Poisson superalgebra then becomes a left one, and vice versa.

In a right Gerstenhaber algebra, $A$ and $L$ are identical as ungraded $k$-modules, but as graded modules the Lie degree is reduced by 1 , so one has

$$
\begin{gathered}
{\left[a^{m}, b^{n}\right]=-(-1)^{(m-1)(n-1)}\left[b^{n}, a^{m}\right]} \\
(-1)^{(m-1)(p-1)}\left[a^{m},\left[b^{n}, c^{p}\right]\right]+(-1)^{(n-1)(m-1)}\left[b^{n},\left[c^{p}, a^{m}\right]\right] \\
+(-1)^{(p-1)(n-1)}\left[c^{p},\left[a^{m}, b^{n}\right]\right]=0 \\
{\left[a^{m} b^{n}, c^{p}\right]=\left[a^{m}, c^{p}\right] b^{n}+(-1)^{m(p-1)} a^{m}\left[b^{n}, c^{p}\right] .}
\end{gathered}
$$


The last equation asserts that right Lie multiplication by $c^{p}$ acts as a graded derivation of degree $p-1$ of the graded associative algebra $A$. In a left Gerstenhaber algebra, it is left Lie multiplication which acts as a graded derivation. As before, if the associative multiplication is replaced by its opposite and the Lie multiplication preserved, then "right" and "left" are interchanged.

Gerstenhaber algebras (and in fact the concept of a graded Lie algebra) first arose in algebraic deformation theory as the Hochschild cohomology $H^{\bullet}(A, A)$ of an associative algebra $A$ with coefficients in itself, [7]. [Under the newer convention which introduces a sign change when an operator passes an argument, $H^{\bullet}(A, A)$ becomes a left Gerstenhaber algebra. Specifically, if $f$ and $g$ are, respectively, $m-$ and $n$-cochains of $A$ with coefficients in itself and $a, b$ respectively $m$ - and $n$-tuples of elements of $A$, then Hochschild originally defined $(f \cup g)(a, b)$ to be $f(a) g(b)$ while the newer convention sets $(f \cup g)(a, b)=(-1)^{m n} f(a) g(b)$ because $g$, which is viewed as having degree $n$, has passed $a$, which has degree $m$.]

Notice that the degree 0 part of a Poisson superalgebra is an ordinary Poisson algebra, but "degree zero part" has less meaning for a Gerstenhaber algebra since what is of even degree for the associative structure is of odd degree for the graded Lie structure, and conversely. Nevertheless it is true that the elements of degree 0 form an associative algebra and those of degree 1 a Lie algebra. (Warning: Some authors unfortunately use "Poisson superalgebra" for both cases.)

\section{A missing EQUivalence}

Even in the ungraded case, the operad approach to the cohomology of Poisson algebras does not lend itself easily to exhibiting the basic relation between cohomology and deformations, and it is not immediately applicable to Leibniz pairs. The latter problem could be overcome if there were an equivalence between the category of Leibniz pairs and a subcategory of the category of associative algebras or even if Leibniz pairs were equivalent to algebras over a certain operad. While Leibniz pairs are definitely not themselves algebras over an operad, it seems likely that the data carried by a Poisson algebra is equivalent to a number of operations satisfying certain identities on a single vector space. But this has so far not been shown. Here we construct a cohomology theory for Leibniz pairs and algebras in an elementary way, as the cohomology of a certain double complex, and show that (as in the classical algebraic deformation theory [8] and that of bialgebras [12, 13, 2]) the first cohomology group contains the infinitesimal automorphisms, the second cohomology group contains the infinitesimal deformations and the third group the obstructions to these. Since the concept of a module over a Leibniz pair is meaningful, as we show in the next section, other elementary (i.e., non-operadic) definitions of cohomology are also possible (e.g., as a Yoneda type Ext, or as a derived functor), but we do not know if all these coincide. While injective and projective modules may be defined in the usual way, we do not know if there exist "enough" injective or projective modules over a 
Leibniz pair $(A, L)$, that is, whether every module is a submodule of an injective or quotient of a projective. (The existence of enough injectives would imply that the standard construction of the cohomology using injective resolutions is possible.)

We do not know either if there is a Gerstenhaber algebra structure on the cohomology of a Leibniz pair with coefficients in itself or even simply a graded Lie structure. In the special case where the Leibniz pair structure is induced from a Lie group operation there is some evidence that the Gerstenhaber algebra structure should exist. We will return to this at the end of the paper.

\section{Modules}

In a Leibniz pair $(A, L)$ we will generally denote the elements of $A$ by $a, b, c, \ldots$ and those of $L$ by $x, y, z, \ldots$ Suppose that $M$ is an $A$-bimodule and $P$ an $L$-module, and denote by $A+M$ and $L+P$, respectively, the associative and Lie semidirect products. Then $(M, P)$ will be called an $(A, L)$ - module if there is a Lie algebra morphism $L+P \rightarrow \operatorname{Der}(A+M)$ extending the structure morphism $\mu: L \rightarrow \operatorname{Der} A$ such that $[L, M],[P, A] \subset M$ and $[P, M]=0$. These are grading conditions: view $L$ and $A$ as of degree zero and $P$ and $M$ as of degree one; then $[P, M]=0$ because there is nothing of degree two. Note that if $\pi \in P$ then the hypotheses imply that the map of $A$ into itself sending $a$ to $[\pi, a]$ is a derivation of $A$ into $M$, so we have a map $P \rightarrow \operatorname{Der}(A, M)$.

Every Leibniz pair $(A, L)$ is a module over itself, but it is helpful to distinguish between $A$ the algebra and $A$ the $A$-bimodule. In this case we may denote the module by $t A$, where $t$ is some variable whose square is zero. Then $A+t A$ is identical with the $k[t] / t^{2}$-module $A[t] / t^{2}$ which, when viewed as a $k$-algebra, is just the semidirect product. We will then similarly write $t L$. If $A$ is a Poisson algebra then a module $M$ over $A$ is really a module $(M, M)$ over the pair $(A, A)$, where the two Lie products of both $M$ and $A$ are identical.

The concept of a morphism of Leibniz pairs is clear, whence so is that of a morphism of modules over a Leibniz pair. One has kernels and cokernels, hence an abelian category, and also direct sums in the algebraic sense, i.e, categorical direct products. The category of Leibniz pairs contains the categories of associative and Lie algebras as full subcategories. (In the first case, take $L=0$, in the second $A=k$.)

\section{The Double COMPlex for A Leibniz PAir}

Unless stated otherwise, all tensor products will be over the coefficient ring $k$. (For much of what follows this need not be of characteristic 0 , but to fix the ideas one may assume that it is $\mathbb{R}$.) Since we do not consider the graded case, we will henceforth denote $\otimes^{p} A$ simply by $A^{p}$. Suppose that $(A, L)$ is a Leibniz pair and that $(M, P)$ is a module over it. Let $C^{p}(A, M)=\operatorname{Hom}_{k}\left(A^{p}, M\right)$, the $p$ th Hochschild cochain group of $A$ with coefficients in $M$. Note that $C^{0}(A, M)$ is just $M$. Now $C^{p}=C^{p}(A, M)$ is 
again an $L$-module in the usual way, where if $f \in C^{p}$ then one sets

$$
[x, f]\left(a_{1}, \ldots, a_{p}\right)=\left[x, f\left(a_{1}, \ldots, a_{p}\right)\right]-\sum_{i=1}^{p} f\left(a_{1}, \ldots,\left[x, a_{i}\right], \ldots, a_{p}\right) .
$$

It is immediate that $\operatorname{Der}(A, M)$ is then an $L$-submodule of $C^{1}(A, M)$, and the mapping $P \rightarrow \operatorname{Der}(A, M)$ noted above is an $L$-module morphism.

The Hochschild coboundary $\delta_{H}: C^{p} \rightarrow C^{p+1}$ is given by

$$
\begin{gathered}
\left(\delta_{H} f\right)\left(a_{1}, \ldots, a_{p+1}\right)=a_{1} f\left(a_{2}, \ldots, a_{p+1}\right)+ \\
\sum_{i=1}^{p}(-1)^{p} f\left(a_{1}, \ldots, a_{i} a_{i+1}, \ldots, a_{p+1}\right)+(-1)^{p+1} f\left(a_{1}, \ldots, a_{p}\right) a_{p+1} .
\end{gathered}
$$

It is an essentially trivial matter to verify the following fundamental result.

Proposition 1. Let $(M, P)$ be a module over a Leibniz pair $(A, L)$. Then $\delta_{H}$ : $C^{p}(A, M) \rightarrow C^{p+1}(A, M)$ is a morphism of Lie modules over $L$.

Let $(M, P)$ be a module over the Leibniz pair $(A, L)$. The double complex $C^{\bullet \bullet \bullet}(A, L ; M, P)$ of $(A, L)$ with coefficients in $(M, P)$ is defined as follows. For all $p>0, q \geq 0$, set $C^{p, q}=\operatorname{Hom}_{k}\left(\wedge^{q} L, C^{p}(A, M)\right) \cong \operatorname{Hom}_{k}\left(A^{p} \otimes \wedge^{q} L, M\right)$, and set $C^{0, q}=\operatorname{Hom}_{k}\left(\wedge^{q} L, P\right)$. (Note that $A^{0}=\wedge^{0} L=k$.) For $p>0$, the "vertical" coboundary $C^{p \cdot q} \rightarrow C^{p+1, q}$ is just the Hochschild coboundary $\delta_{H}$ (the index $h$ stands for Hochschild, not for horizontal!). For $p=0$ note that we are given a Lie module $\operatorname{Hom}_{k} A=C^{1}(A, M)$. This induces, for every $q$, the desired map $C^{0, q} \rightarrow C^{1, q}$ which we denote $\delta_{v}$. The composition of two vertical coboundary operators $C^{p, q} \rightarrow C^{p+2, q}$ is zero for all $p$ and $q$ since for $p>0$ both are Hochschild coboundaries, while for $p=0$ it follows because $\operatorname{Der}(A, M)$ is precisely the kernel of $\delta_{H}: C^{1}(A, M) \rightarrow C^{2}(A, M)$.



In the horizontal direction we have for all $p$ and $q$ the Chevalley-Eilenberg coboundary $\delta_{C E}: C^{p, q} \rightarrow C^{p, q+1}$. Since all the vertical coboundaries are Lie module morphisms, it follows that they commute with all the horizontal ones. Denoting any 
vertical coboundary for the moment by $\delta_{H}$ we can therefore define a total cohomology complex with $C_{t o t}^{n}=\bigoplus_{p+q=n} C^{p, q}$ and $\delta_{t o t}: C_{t o t}^{n} \rightarrow C_{t o t}^{n+1}$ whose restriction to $C^{p, q}$ by definition is $\delta_{H}+(-1)^{p} \delta_{C E}$. Its cohomology we define to be the cohomology of the Leibniz pair $(A, L)$ with coefficients in the module $(M, P)$, denoted $H^{\bullet}(A, L ; M, P)$. When we consider the cohomology of $(A, L)$ with coefficients in itself as a module, we will write simply $C^{p, q}(A, L)$ and $H^{\bullet}(A, L)$.

Denote the invariants of an $L$-module $N$, i.e., the set of all $n \in N$ with $[x, n]=0$ for all $x \in L$ by $N^{L}$. In the double complex $C^{\bullet, \bullet}(A, L ; M, P)$ the kernel of $\delta_{C E}$ : $C^{p, 0} \rightarrow C^{p, 1}$ is just $C^{p}(A, M)^{L}$ for all $p>0$, and for $p=0$ it is $(P)^{L}$. (When cohomology is taken with coefficients in $(A, L)$ itself this will be the center $Z(L)$ of $L$.) The "augmenting column" of the double complex is therefore the complex

$$
C^{\bullet}: P^{L} \rightarrow C^{1}(A, M)^{L} \rightarrow C^{2}(A, M)^{L} \rightarrow \ldots
$$

The first arrow is induced by the morphism $P \rightarrow \operatorname{Der}(A, M)$ (which, when cohomology is taken with coefficients in $(A, L)$ is the structural morphism $\mu: L \rightarrow$ Der $A$ ) and all the others by the Hochschild coboundary. Note that $H^{i}(A, L ; M, P)$ is in particular an $L$ - module and is finite-dimensional if all the algebras and modules are.

While the cohomology groups of the double complex are not easy to compute, the Whitehead lemmas and the fact that the cohomology of $L$ with coefficients in itself vanishes give the following

Theorem 2. Suppose that $(M, P)$ is a module over a Leibniz pair $(A, L)$ with all algebras and modules finite-dimensional over the real or complex numbers and $L$ semisimple. Then $H^{i}(A, L ; M, P)$ is naturally isomorphic to $H^{i}\left(C^{\bullet}\right)$ for $i=1,2,3$.

If the associative algebra $A$ of a Leibniz pair is commutative, and if the coefficient ring $k$ has characteristic 0 , that is, if there is a unital morphism $\mathbb{Q} \rightarrow k$ then each of the $C^{p}(A, M)$ decomposes into its Hodge parts [10] and one may check that this decomposition is preserved by the Chevalley-Eilenberg coboundary. The entire double complex therefore has a Hodge decomposition, the first component of which is obtained by replacing each $C^{p}(A, M)$ by its submodule of Harrison cochains (cochains vanishing on shuffles). (The augmenting column similarly decomposes, giving the augmenting columns of the Hodge components.)

\section{The double complex for a Poisson Algebra}

When $A$ is a (not necessarily commutative) Poisson algebra, the Lie module operation of $A$ on itself can not be deformed independently of the Lie multiplication on $A$, so the foregoing double complex must be modified by the elision of one row. In this section we will assume characteristic zero, but for the deformation theory it is really only necessary to assume the invertibility of 2 and 3 in $k$.

Let $M$ be a Poisson $A$-module, i.e., simultaneously an associative $A$-bimodule and a Lie $A$-module where for each fixed $m \in M$ the mapping sending $a \in A$ 
to $[a, m] \in M$ is a derivation of $A$ into $M$. Note that we do not have to assume separately that there is given a map $M \rightarrow C^{1}(A, M)$; for this we simply take the usual Hochschild coboundary in which $\left(\delta_{H} m\right)(a)=a m-m a$. (This can vanish identically, in which case $M$ is called a "symmetric" module. A particular case is that in which $A$ is a commutative Poisson algebra and $M$ is $A$ itself.) We will define a double complex $\widetilde{C}^{\bullet \bullet \bullet}(A ; M)$ with $\widetilde{C}^{p, q}=C^{p, q}=\operatorname{Hom}_{k}\left(\left(A^{p}\right) \otimes\left(\wedge^{q} A\right), M\right)$ for $p \geq 2$, but with $\widetilde{C}^{1, q}=\operatorname{Hom}_{k}\left(\wedge^{q+1} A, M\right)$ and $\widetilde{C}^{0, q}$ undefined. The lowest vertical coboundary, from $\widetilde{C}^{1, q}$ to $\widetilde{C}^{2, q}$, will be denoted $\delta_{P}$, (the "Poisson" coboundary).

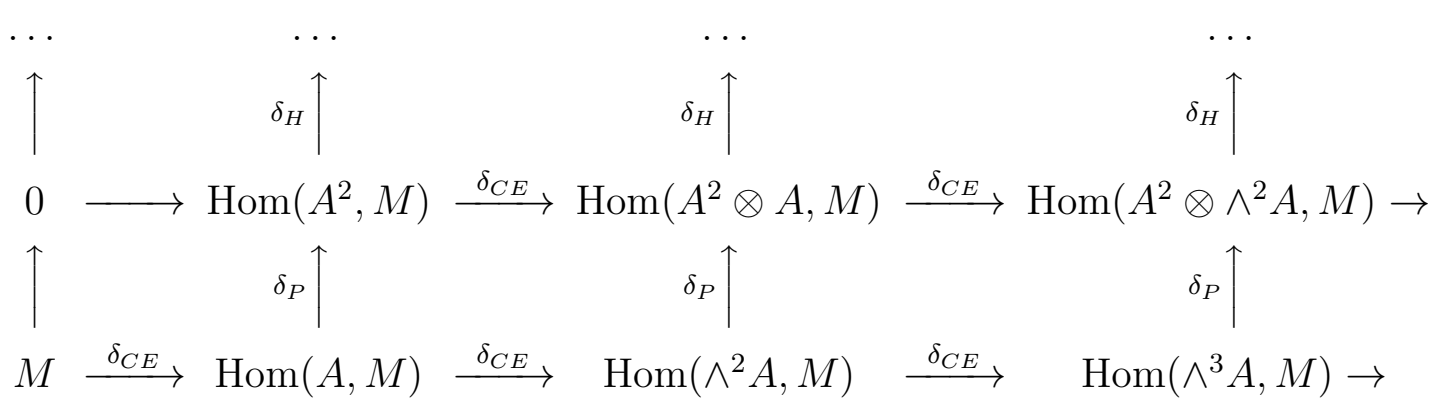

Suppose now that $V$ is simply a $k$-module and denote $\otimes^{p} V$ by $V^{p}$. We view $\bigwedge^{p} V$ as the submodule of $V^{p}$ consisting of all skew elements and denote by $\epsilon_{p}: V^{p} \rightarrow$ $\wedge^{p} V$ the projection sending $v_{1} \otimes \cdots \otimes v_{n}$ to $v_{1} \wedge \cdots \wedge v_{n}$. If $a \in V^{p}, b \in V^{q}$ then $\epsilon_{p+q} a \otimes b=\epsilon_{p+q}\left(\epsilon_{p} a \otimes \epsilon_{q} b\right)$. We will henceforth write $\epsilon$ without the subscript. Let $\epsilon^{*}$ be the dual map $\operatorname{Hom}_{k}\left(\wedge^{q} A, M\right) \rightarrow \operatorname{Hom}_{k}\left(A \otimes\left(\wedge^{q-1} A\right), M\right)$. If $f$ is in the latter module, we will write $f\left(a_{1} \mid a_{2} \wedge \cdots \wedge a_{q}\right)$ for its value at $a_{1} \otimes\left(a_{2} \wedge \cdots \wedge a_{q}\right)$. Now let $\delta_{P}:\left(\bigwedge^{q} A, M\right) \rightarrow\left(A^{2} \otimes \wedge A^{q-1}\right)$ be the composite of $\epsilon^{*}$ with $\delta_{H}: \operatorname{Hom}_{k}(A \otimes$ $\left.\bigwedge^{q-1} A, M\right) \rightarrow \operatorname{Hom}_{k}\left(A^{2} \otimes \bigwedge^{q-1} A, M\right)$, and view it as a "vertical" coboundary. (Note that this is vertical by virtue of our renumbering, but that it is really our original row 1 which is missing.)

Proposition 3. In the original double complex, if $f \in C^{0, q}$ then $\delta_{C E} \epsilon^{*} f+\epsilon^{*} \delta_{C E} f$ $=\delta_{v} f$.

Proof. From the definitions we have

$$
\begin{gathered}
\left(\delta_{C E} f\right)\left(a_{1} \wedge \cdots \wedge a_{q+1}\right)=\sum_{i=1}^{q+1}(-1)^{i}\left[a_{i}, f\left(a_{1} \wedge \cdots \wedge \hat{a}_{i} \wedge \cdots\right)\right]+ \\
\sum_{j>i}(-1)^{i+j} f\left(\left[a_{i}, a_{j}\right] \wedge \cdots \hat{a}_{i} \cdots \hat{a}_{j} \cdots\right)
\end{gathered}
$$


while

$$
\begin{aligned}
\left(\delta_{C E} \epsilon^{*} f\right)\left(a_{1} \mid a_{2} \wedge\right. & \left.\cdots \wedge a_{q+1}\right)=\sum_{i=2}^{q+1}(-1)^{i}\left[a_{i},\left(\epsilon^{*} f\right)\left(a_{1} \mid a_{2} \wedge \cdots \wedge \hat{a}_{i} \wedge \cdots a_{q+1}\right)\right]+ \\
& \sum_{j>i \geq 2}(-1)^{i+j}\left(\epsilon^{*} f\right)\left(a_{1} \mid\left[a_{i}, a_{j}\right] \wedge \cdots \hat{a}_{i} \cdots \hat{a}_{j} \cdots\right) .
\end{aligned}
$$

It follows immediately that

$$
\begin{gathered}
\left(\epsilon^{*} \delta_{C E} f\right)\left(a_{1} \mid a_{2} \wedge \cdots \wedge a_{q+1}\right)= \\
-\left(\delta_{C E} \epsilon^{*} f\right)\left(\left(a_{1} \mid a_{2} \wedge \cdots \wedge a_{q+1}\right)+\right. \\
{\left[a_{1}, f\left(a_{2} \wedge \cdots a_{q+1}\right)\right]+\sum_{j=2}^{q+1}(-1)^{1+j} f\left(\left[a_{i}, a_{j}\right] \mid a_{2} \wedge \cdots \hat{a}_{j} \wedge \cdots a_{q+1}\right)}
\end{gathered}
$$

which implies the formula.

It follows, in particular, that in a commutative Poisson algebra if $f \in C^{0, q}$ then $\delta_{C E} \epsilon^{*} f+\epsilon^{*} \delta_{C E} f=0$. Note, incidentally, the similarity of the result with the classical formula in differential geometry $d i+i d=\mathcal{L}$.

Theorem 4. If $f \in C^{0, q}$ then

$$
\delta_{C E} \delta_{P} f+\delta_{P} \delta_{C E} f=0 .
$$

Proof. Apply $\delta_{H}$ to both sides of the foregoing formula and note that $\delta_{H} \delta_{v}=0$, while $\delta_{H}$ and $\delta_{C E}$ commute.

It follows that we indeed have a double complex. (Since the horizontal and vertical coboundaries in our original complex commute, one should really replace the horiziontal coboundary on the $q$-th column with $(-1)^{q} \delta_{C E}$ so that they now anti-commute. The total coboundary is then just the sum of horizontal and vertical coboundaries and $\delta_{P}$ is now the appropriate first vertical coboundary in the modified double complex.) There is no evident relation between the cohomology of the double complex for a Leibniz pair and that for a Poisson algebra. In particular, neither is included into nor a quotient of the other. Generally, when the bottom rows of a double complex are altered, the total cohomology groups may change in unknown ways.

\section{Deformation of Leibniz pairs and Poisson algebras}

The deformation theory of a Leibniz pair now parallels that of an associative algebra $([8])$, the controlling cohomology being that of the pair with coefficients in itself.

When a Leibniz pair $(A, L)$ is given we will, as before, generally denote the elements of $A$ by $a, b, \ldots$ and of $L$ by $x, y, \ldots$ Let $\alpha: A \times A \rightarrow A$ denote the associative multiplication map, $\lambda: L \times L \rightarrow L$ be the Lie multiplication, and write $\mu(x)(a)=$ 
$\mu(x, a)$, viewing $\mu$ as a map $L \times A \rightarrow A$. The Leibniz pair may then be denoted $(\alpha, \mu, \lambda)$. By a deformation of this Leibniz pair we will mean a Poisson pair of the form $\left(\alpha_{t}, \mu_{t}, \lambda_{t}\right)$ where $\alpha_{t}$ is a deformation of $\alpha(c f .[8])$, i.e., an associative $k[[t]]-$ bilinear multiplication $A[[t]] \times A[[t]] \rightarrow A[[t]]$ expressible as a power series $\alpha_{t}(a, b)=$ $a b+t \alpha_{1}(a, b)+t^{2} \alpha_{2}(a, b)+\ldots$ where each $\alpha_{i}$ is a bilinear map $A \times A \rightarrow A$ extended to be a $k[[t]]$-bilinear map $A[[t]] \times A[[t]] \rightarrow A[[t]]$, and similarly for $\lambda_{t}, \mu_{t}$. The triple $\left(\alpha_{1}, \mu_{1}, \lambda_{1}\right)$ of first order terms is the infinitesimal of the deformation. Note that as $\lambda_{t}$ must be skew, so are all the $\lambda_{i}$ and in particular $\lambda_{1}$. It is clear that $\alpha_{1}$ must be an infinitesimal deformation of $A$ as an associative algebra, i.e., a Hochschild 2-cocycle of $A$ with coefficients in $A$ itself, and that $\lambda_{1}$ is an infinitesimal deformation of $L$, and so a 2-cocycle in the Chevalley-Eilenberg theory of $L$ with coefficients in $L$. In addition, there are compatibility conditions betweeen $\alpha_{1}$ and $\mu_{1}$ and between $\mu_{1}$ and $\lambda_{1}$ which one obtains from the linear terms in the following equations, respectively:

$$
\begin{aligned}
& \mu_{t}\left(x, \alpha_{t}(a, b)\right)=\alpha_{t}\left(a, \mu_{t}(x, b)\right)+\alpha_{t}\left(\mu_{t}(a, a), b\right), \\
& \mu_{t}\left(\lambda_{t}(x, y), a\right)=\lambda_{t}\left(x, \mu_{t}(y, a)\right)-\mu_{t}\left(y, \mu_{t}(x, a)\right)
\end{aligned}
$$

These, when written out together with the foregoing, state precisely that $\left(\alpha_{1}, \mu_{1}, \lambda_{1}\right)$ constitute a 2-cocycle of the total cohomology arising from the double complex $C^{\bullet} \bullet(A, L ; A, L)$. (The idea of the double complex we have constructed is borrowed, in spirit, from the construction of the cohomology of a bialgebra, cf. [12], or for a better exposition [13].)

There is a natural concept of equivalence of deformations: we say that $\left(\alpha_{t}, \mu_{t}, \lambda_{t}\right)$ is equivalent to $\left(\alpha_{t}^{\prime}, \mu_{t}^{\prime}, \lambda_{t}^{\prime}\right)$ where $\alpha_{t}^{\prime}=\alpha+t \alpha_{1}^{\prime}+t^{2} \alpha_{2}^{\prime}+\ldots$, etc., if there exist $k[[t]]$-linear maps $\Phi_{t}=(i d)_{A}+t \phi_{1}+t^{2} \phi_{2}+\cdots: A[[t]] \rightarrow A[[t]]$ (with each $\phi_{i}$ a linear map $A \rightarrow A$ extended to be $k[[t]]$-linear) and $\Psi_{t}=\operatorname{id}_{L}+t \psi_{1}+t^{2} \psi_{2}+\cdots$ : $L[[t]] \rightarrow L[[t]]$ such that

$$
\begin{aligned}
& \alpha_{t}^{\prime}(a, b)=\Phi_{t}^{-1} \alpha_{t}\left(\Phi_{t} a, \Phi_{t} b\right), \\
& \mu_{t}^{\prime}(x, a)=\Phi_{t}^{-1} \mu_{t}\left(\Psi_{t} x, \Phi_{t} a\right), \\
& \lambda_{t}^{\prime}(x, y)=\Psi_{t}^{-1} \lambda_{t}\left(\Psi_{t} x, \Psi_{t} y\right) .
\end{aligned}
$$

As expected, equivalent deformations have cohomologous infinitesimals, and any cocycle in the cohomology class of the infinitesimal of a deformation can be taken as the infinitesimal of an equivalent deformation. Up to equivalence, therefore, only the cohomology class of an infinitesimal is of consequence. Obstructions arise (at least in principle) exactly as in earlier deformation theories: if $\left(\alpha_{t}, \mu_{t}, \lambda_{t}\right)$ are given through terms in $t^{n-1}$ and satisfy the definition of a Leibniz pair modulo $t^{n}$, then there is generally an obstruction in the third cohomology group to extending through terms in $t^{n}$ in such a way that the conditions for a Leibniz pair are satisfied modulo $t^{n+1}$. 
In the subcategory of Poisson algebras by definition we have $A=L$ and $\mu=\lambda$, and in the foregoing must take $\Psi_{t}=\Phi_{t}$. We then must use the double complex $\widetilde{C}^{\bullet \bullet \bullet}(A ; A)$ and have analogous assertions to the foregoing. Restriction to the subcategory of commutative Poisson algebras is then essentially that of the passage from Hochschild to Harrison cohomology.

In addition to deformations, one-parameter families of automorphisms (restricted to be the identity at $t=0$ ) were considered in [8]. There an infinitesimal automorphism of an algebra $A$ was simply a derivation, and in characteristic zero there was no formal obstruction to finding a family with this infinitesimal since one could exponentiate the derivation. The matter is less simple for a Leibniz pair $(A, L)$ since an infinitesimal automorphism is now a pair $(\phi, \psi)$ constituting a one-cocycle of our double complex and it appears that even in characteristic zero there is an obstruction to constructing the higher order terms. For a Poisson algebra $A$, an infinitesimal automorphism is any $\phi: A \rightarrow A$ which is simultaneously a derivation of both the associative and Lie structures, and these can be formally exponentiated. Amongst them are all ad $a$ for $a \in A$.

Recall that when an ordinary associative algebra $A$ with multiplication $\alpha$ is deformed to $A_{t}$, the new multiplication being $\alpha_{t}$, then it is not always possible to "lift" or extend a cocycle $F$ of $A$ (with coefficients in itself) to one of $A_{t}$, cf. [9]. The primary obstruction is the class of the graded Lie product $\left[F, \alpha_{1}\right]$. But even if every derivation could be lifted, there might be obstructions in the sense of the present paper. For note that when $(A, \operatorname{Der} A)$ is a Leibniz pair, a deformation requires that the Lie algebra structure of Der $A$ actually be deformed. By contrast, if $A$ alone is deformed and every element of Der $A$ is liftable, it is still conceivable that the Lie algebra which they generate is now of greater dimension than originally. Also, recall that when a non-nilpotent Lie group $G$ operates on an associative algebra $A$, then the various universal deformation formulas based on the group (cf. [3]) generally produce deformations of $A$ which no longer permit the operation of the full group $G$; symmetry is generally broken. Here, however, we are in particular investigating ways in which $A$ deforms while preserving the full operation of a Lie group (although the operation itself may simultaneously be deformed).

When $A$ is finite-dimensional, one gets some information about the deformation problem from a theorem of Kubo, [17]. First, $A$ is said to be $L$-simple, if it contains no proper ideal which is simultaneously an $L$-submodule. Kubo's theorem asserts that such $A$ must be a total matric algebra, that is, the algebra of all $n \times n$-matrices for some $n$, or multiplication in $A$ must be identically zero and $A$ must be nothing more than an irreducible $L$-module, made into an algebra with the trivial multiplication. When $L$ is simple, there can be no deformations of this structure, but for general $L$ the question becomes one of the deformation theory of modules over a Lie algebra. 


\section{Afterword: Application of the Cohomology Comparison Theorem}

For a presheaf of algebras over a small category, one can define in a natural way a cohomology theory which, remarkably, is identical with the cohomology of a single ring built from the presheaf; this is part of the "Cohomology Comparison Theorem" (CCT) of [11]. (One consequence is that for every simplicial complex there is an algebra whose cohomology with coefficients in itself is naturally isomorphic to the simplicial cohomology of the complex.) Since the cohomology has been reduced to that of a single ring, it follows from the original results of [7] that there indeed exists a Gerstenhaber algebra structure on the cohomology of the original presheaf. However, while for a single algebra $A$ there is already a graded Lie structure on the Hochschild cochains of $A$ with coefficients in itself, this is not the case for a presheaf of algebras, where the graded Lie structure exists only on the cohomology level. It is therefore far from transparent. Nevertheless, it suggests that when we have a Leibniz pair $(A, L)$ induced from the action of a Lie group on $A$ then we should apply the CCT to the group $G$ (considered as a small category) and the presheaf which to the unique object of this category assigns the algebra $A$. The essential thing we do not know is what relation the cohomology so obtained has to that which we have just defined for the pair $(A, L)$. If these turn out to be the same, then we shall have, amongst other things, that there is a Gerstenhaber algebra structure on the cohomology of $(A, L)$. This approach may be applicable to the case where the given Leibniz pair consists of the smooth functions and smooth vector fields on a compact manifold. If it is, then one would expect that the Gerstenhaber algebra structure is given by explicit (although difficult) formulas whose nature really should not depend on the fact that we started with a compact manifold. It would seem, therefore, that there is at least some hope of exhibiting a Gerstenhaber algebra structure on the cohomology of a Leibniz pair or Poisson algebra.

Acknowledgment. We are grateful to Y. Kosmann-Schwarzbach, F. Kubo, M. Markl, J. Stasheff, D. Sternheimer, A. Weinstein, and P. Xu for helpful discussions.

\section{REFERENCES}

1. F. Bayen, M. Flato, C. Fronsdal, A. Lichnerowicz and D. Sternheimer, Deformation theory and quantization, I and II, Ann. Phys. (NY), 111 (1978), 61-151.

2. P. Bonneau, M. Flato, M. Gerstenhaber and G. Pinczon, The hidden group structure of quantum groups: strong duality, rigidity and preferred deformations, Commun. Math. Phys. 161 (1994), $125-156$.

3. V. Coll, M. Gerstenhaber, and S. D. Schack, Universal deformation formulas, J. Pure and Appl. Alg. 90 (1993), 201-219.

4. A. Connes, Non-commutative differential geometry, Publ. Math. IHES 62 (1986), 41-144. Géométrie Non-Commutative, Interéditions, Paris (1990) [expanded English edition, preprint IHES/M/93/12, March 1993, to be published by Academic Press].

5. A. Connes, M. Flato and D. Sternheimer, Closed star-products and cyclic cohomology, Lett. Math. Phys. 24 (1992), 1-12. 
6. T. Fox and M. Markl, Distributive laws and the cohomology, Preprint, University of North Carolina, Chapel Hill, 1994.

7. M. Gerstenhaber, The cohomology structure of an associative ring, Ann. of Math. 78 (1963), $267-288$.

8. _ On the deformation of rings and algebras, Ann. of Math. 79 (1964), 59-103.

9. . On the deformation of rings and algebras: IV, Ann. of Math. 99 (1974), 257-276.

10. M. Gerstenhaber and S. D. Schack, A Hodge-type decomposition for commutative algebra cohomology, J. Pure and Appl. Alg. 48 (1987), 229-247.

11. _ Algebraic cohomology and deformation theory, In: Deformation Theory of Algebras and Structures and Applications (M. Hazewinkel and M. Gerstenhaber, eds.), Kluwer Academic Publishers, 1988, pp. 11-264.

12. B Bialgebra cohomology, deformations, and quantum groups, Proc. Nat. Acad. Sci. (U.S.A.) 87 (1990), 478-481.

13. _ Algebras, bialgebras, quantum groups, and algebraic deformations, In: Deformation Theory and Quantum Groups with Applications to Mathematical Physics (Providence) (M. Gerstenhaber and J. Stasheff, eds.), Contemporary Math., vol. 134, Amer. Math. Soc., Providence, 1992, pp. 51-92.

14. E. Getzler and J. D. S. Jones, Operads, homotopy algebra and iterated integrals for double loop spaces, Preprint, Department of Mathematics, MIT, March 1994, hep-th/9403055.

15. V. Ginzburg and M. Kapranov, Koszul duality for operads, Duke Math. J. 76 (1994), 203-272.

16. Y. Kosmann-Schwarzbach and F. Magri, Poisson-Nijenhuis structures, Ann. Inst. Henri Poincaré, vol.53(1), (1990), 35-81.

17. F. Kubo, The triviality of the associative products on finite-dimensional semisimple Poisson algebras, Preprint, University of Pennsylvania, December 1994.

18. R. S. Palais, The cohomology of Lie rings, Proc. Symp. Pure Math. 3, Amer. Math. Soc. (1961), 130-137.

19. G. S. Rinehart, Differential forms on general commutative algebras, Trans. Amer. Math. Soc. 108 (1963), 195-222.

20. I. Vaisman, Lectures on Poisson manifolds, Progress in Mathematics 118, Birkhaüser (1994).

21. P. Xu, Non-commutative Poisson algebras, Amer. J. Math. 116 (1994), 101-125.

Physique Mathématique, Université de Bourgogne, B.P. 138, F-21004 Dijon Cedex, FRANCE

E-mail address: flato@satie.u-bourgogne.fr

Department of Mathematics, University of Pennsylvania, Philadelphia, PA 191046395, USA

E-mail address: mgersten@mail.sas.upenn.edu or murray@math.upenn.edu (TeX files)

Department of Mathematics, University of Pennsylvania, Philadelphia, PA 191046395, USA

E-mail address: voronov@math.upenn.edu 